\title{
Intelligent Space for Building Fire Detection and Evacuation Decision Support
}

\author{
Z.C. Zou \\ HIT-UCB/IURD Joint Research Centre of Sustainable Urban \\ Development \\ School of Architecture \\ Harbin Institute of Technology \\ China \\ K.L. Wu \\ HIT-UCB/IURD Joint Research Centre of Sustainable Urban \\ Development \\ School of Architecture \\ Harbin Institute of Technology \\ China
}

\begin{abstract}
Building fires cause incredible life and property losses. In order to enhance buildings safety a framework of building up intelligent space based fire detection and evacuation system is clarified in this paper. On the base of building fire defence analysis, this paper illustrates the application of intelligent space with functionalities of intelligent building fire detection and emergency evacuation spatial decision support. Geo-spatial data mining approach (SPDA) has been used to design the artificial intelligent module of fire sensors to improve their ability of fire detection. And a web-GIS based spatial decision support system can provide dynamic routing service for evacuees and emergency managers. The intelligent space provides reliable fire alarm detection, effective fire suppression, and adaptable evacuation strategies for occupants, which will enhance evacuees' survival possibility in building fires.
\end{abstract}

Keywords-intelligent space; building fire evacuation; spatial decision support system

\section{INTRODUCTION}

Rapid urbanization in China makes cities become crowed and complex. Large-scale buildings, super high rise buildings and huge underground constructions bring more fire safety problems. Building Fires present an enormous cost of human life, livelihood and properties. Deaths and injuries occurs when people cannot escape fires (Lee, 2013).

Substantial interruption due to false alarms may occur as the area where alarm systems are vacated and thoroughly checked before work is resumed (Gupta and Dharmadhikari, 1985). Due to dynamic fire propagation in buildings, the suppression systems (usually water mist or halogenated agents) may threat evacuees (Mowrer and Pecht, 1995).

According to an extensive analysis of fatalities and injuries in building fires (Hasofer and Thomas, 2006), "condition preventing escape" is the first reason to casualties, and "no time to escape" is the second. As "fire between casualty and

\author{
H. Leng \\ Department of Urban Planning \\ School of Architecture \\ Harbin Institute of Technology \\ China
}

W.Q. Su

Department of Urban Planning

School of Architecture

Harbin Institute of Technology

China

exit" can also be a consequence of the fire detection is too late. It is clear that fire detection should be as early as possible. If the building fire prevention system fails damages should be minimized. A proper evacuation is crucially important in such cases (Gupta and Yadav, 2004; Pires, 2005).

When people are under stress (in fires), their behaviours can be unpredictable (Kobes, et al. 2010). It was shown that people prefer to use their familiar ordinary exits even if the distances are longer than the nearest one (Benthorn and Frantzich, 1999). This might be one of the reasons for the high influence of "no time to escape" condition. If we increase the possibility that there might be a fire in a certain path to exit, two most influential "conditions preventing escape" are related to evacuation. That means in building fire emergencies people guidance system are valuable.

\section{FIRE DEFENCE SYSTEMS IN BUILDINGS}

All buildings are designed with active fire defence systems and passive fire defence systems for fire emergencies. And the building fire defence systems perform high efficiency of fire suppression and human life safeguarding. Human behaviours are alternating and complex.

\section{A. Active fire Defence Systems}

Fire detectors are small devices that sense the intrusion of combustion products. Detectors are designed to sense heat, flame, smoke, or other products of combustion. Performance depends on their location relative to the fire, the movement of the combustion products, and detector sensitivity. Detection and alarm services have several uses. The most common one is to sound an alarm or other signals intended to alert occupants in fire emergencies. The second is transmitting signals to a definite location (such as a fire station). The third operation is triggering activation of other fire defences such as closing doors, automatic fire suppression, or smoke control systems. 


\section{B. $\quad$ Passive Fire Defence System}

Buildings can provide many special features that help in fire suppression, egress, and emergency operations. Changing an air-handling system into an emergency smoke management mode is becoming a common feature in many building designs. A design may integrate a variety of other features that can enhance life safety features. For example, emergency lighting and signage can help occupants and fire fighters to move through the building with a better sense of direction and confidence. Communication systems for alerting occupants and providing emergency information are available. Detection instruments can trigger the closing of selected doors held open by magnetic devices and the shutting of dampers in air ducts. Reliability can be enhanced by using emergency electrical systems that operate when the building's normal power supply is interrupted.

\section{INTELLIGENT BUILDING SPACE FRAMEWORK}

The concept of intelligent space was proposed in 1995 by Hashimoto Lab. of University of Tokyo (Lee and Hashimoto, 1995). The first intelligent space was defined as rooms or areas, which are equipped with sensors which enable them to perceive what is happening in them (Appenzeller et al, 1997). With an ongoing development the definition has been extended. Besides being capable of perceiving what is happening, state of the art intelligent spaces should also understand what is happening in and around them. They are also able to build their own models, to communicate with their inhabitants and to act on the basis of decisions they make (Lee and Hashimoto, 2003). The ability of the environment to act as a context-sensitive user interface and to react in certain situations promises a range of application scenarios, such as intelligent hospital, offices, factories, nurse rooms for the aged, etc. Intelligent spaces are usually equipped with active and passive cameras, tactile sensors, interactive apps, wireless devices such as smart phones and pads, and wireless information publishing platforms based on Web-Spatial Decision Support System (W-SDSS). Information perceived by intelligent space is to be provided to the occupants and emergency decision makers as well. General devices distribution framework of building fire evacuation intelligent space of this research is shown as Figure 1.

\section{INTELLIGENT FIRE DETECTION AND AlARM SYSTEM}

\section{A. Fire And Smoke Sensors Fault}

Common fire detection sensors detect the presence of fire based on smoke or gas. Some studies show that they are neither reliable nor fast (Derbel, 2004). The basic problems with these types of sensor are presented in Figure 2.

The response time of the sensor can be improved if the threshold is lower as shown in Figure 2. A lot of research has been done in order to improve the sensor performance from the reliability and response speed point of view. Gas detectors for example show better performance than smoke detectors, because during the initial fire phase, gases are transported more rapidly than smoke particles. From reliability point of view the detection of smouldering fires is one of the main problems. It is namely difficult to distinct them from false fire and real fire.

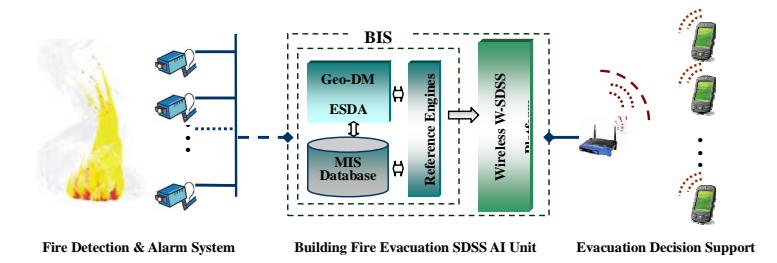

FIGURE I. BUILDING FIRE EVACUATION INTELLIGENT SPACE STRUCTURE

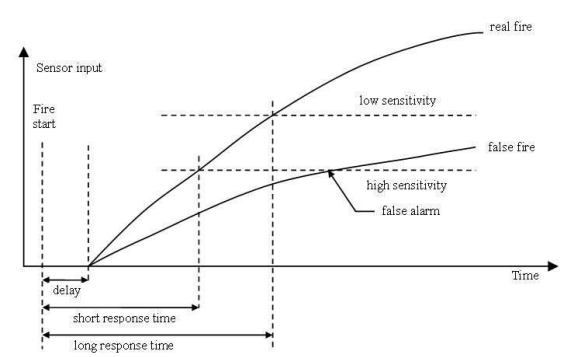

FIGURE II. FIRE AND SMOKE SENSORS’ FAULT

\section{B. Distinguishable Fire Alarm Algorithm}

In order to distinguish false fire and real fire alarm, we employ spatial data mining method to discover images captured by video camera. And set up comparable association rules with other different kinds of sensors (such as smoke/gas sensors, temperature sensors, etc.) to confirm the build fire actual happening.

The Spatial Pattern Discovery Algorithm (SPDA) has been proposed to deal with all these challenges in descriptive task of association rules discovery from spatial data. The multilevel spatial associative classification problem can be formalized as follows:

Given a spatial database (SDB), a set $S$ of reference spatial objects tagged with a class label $y \in\left\{c_{1}, \cdots, c_{L}\right\}$, some sets $R_{k}, 1$ $\leqslant k \leqslant m$ of task-relevant spatial objects, a background knowledge $B K$ including spatial hierarchies $H_{k}$ on objects in $R_{k}, M$ granularity levels in the descriptions ( 1 is the highest while $M$ is the lowest), a set of granularity $\psi_{k}$ which associate each object in $H_{k}$ with a granularity level to deal with several hierarchies at once, a couple of thresholds minsup $[l]$ and minconf $[l]$ for each granularity level, a language bias $L B$ which constrains the search space. For each granularity level:

(1) a set of strong spatial association rules capturing associations between the properties of reference objects and the corresponding class labels $y$;

(2) a classifier to predict $y$ for reference objects.

Spatial association rules are extracted in the form $A \Rightarrow C(s, c)$, where $A$ (antecedent) and $C$ (consequent) are sets of items with $A \cap C=\varnothing$. The support $s$ estimates the probability $p(A \cap C)$, while the confidence $c$ estimates the probability $p(C \mid A)$. The conjunction of literals in $A \cup C$ is 
named spatial pattern since the relations and the objects involved in this pattern have a spatial nature.

The basic idea of SPDA is that a spatial database boils down to a deductive relational database once the spatial relations between reference objects and task-relevant objects have been extracted. For instance, spatial intersection between objects is represented in a derived relation int $\operatorname{er} \sec t s(X, Y)$, $D(S)$ is obtained by augmenting the user supplied $B K$ with the data extracted from SDB and concerns both the reference $(S)$ and task-relevant object $\left\{R_{k}\right\}$. The ground facts in $D(S)$ can be grouped into distinct (not necessarily disjoint) subsets. Each group $O[s]$ is uniquely identified by a reference object $s \in S$ and corresponds to the description of a spatial unit of analysis. The uniqueness of the reference object associated to a spatial unit allow us to well define both the support and confidence of a spatial association rule. More precisely, the spatial association rule: $A \Rightarrow C(s \%, c \%)$, means that in $s \%$ of spatial units of analysis ,both conjunctions $A$ and $C$ hold and in $c \%$ of spatial units of analysis where $A$ is true, also $C$ holds.

Once large patterns have been generated, it is possible to generate strong spatial association rules. For each pattern $P$, SPDA generates antecedents suitable for rules being derived from $P$. The consequent corresponding to an antecedent is simply obtained as the complement of atoms in $P$ and not in the antecedent. Rule constraints are used to specify literals which should occur in the antecedent or consequent of discovered rules. In a more recent release of SPDA, new pattern (rule) constraints have been introduced in order to specify exactly both the minimum and maximum number of occurrences for a literal in pattern (antecedent or consequent of a rule).

\section{Fire Evacuation SPatial Decision SUPPORT}

Intelligent space differs from common fire evacuation procedures mainly because it is situation adaptable. It can namely guide people out of the building along the route that takes the least time. In order to do that, the intelligent space must be able to determine which parts of the building are under fire, and provides reliable routing decision support information for occupants' evacuation.

Most of the real world spatial problems give rise to GIS based multi-criteria analysis. Spatial multi-criteria analysis requires information on criterion values and the geographical distribution of alternatives. The graphical representation of GIS based AHP model is shown in Figure 3.

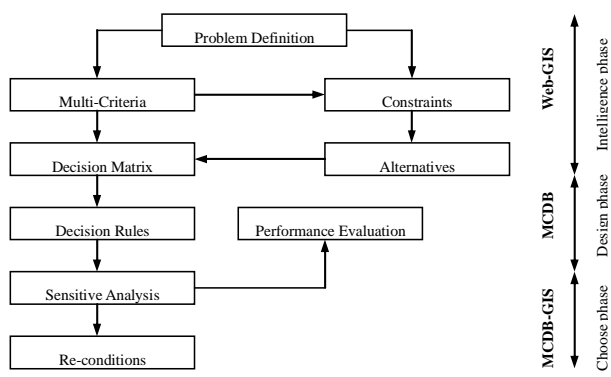

FIGURE III. WEB-GIS AHP MODEL STRUCTURE
The geographical location in GIS data format is represented by line, point or polygons (in vector data models) and by pixel or set of pixels (in raster data model). Therefore, in the spatial decision problem, listing the alternatives is quite difficult because the number of the alternatives are very large. Here we assume the alternatives in building fire evacuation can be presented as a set of variables: $A_{1}=\left\{a_{11}, a_{12}, \ldots, a_{1 m}\right\}$; $A_{2}=\left\{a_{21}, a_{22}, \ldots, a_{2 m}\right\} ; \ldots ; A_{n}=\left\{a_{n 1}, a_{n 2}, \ldots, a_{n m}\right\}$.

A constraint map displays the limitations on the value that attributes and decision variables may assume. The evaluation criteria maps are also referred to as attribute maps, data layers and thematic maps, etc. in GIS terminology. Here we assume that a set of certain criteria could be used in building fire evacuation management decision making $B=\left\{B_{1}, B_{2}, \cdots, B_{n}\right\}$. Tree structure of building fire evacuation decision could be described in Figure 4.

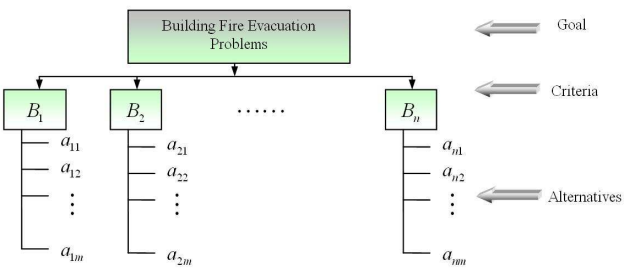

FIGURE IV. INTELLIGENT SPACE BUILDING FIRE EVACUATION DECISION TREE

\section{CONCLUSION}

The intelligent space could be used to improve fire detection, fire suppression, and evacuation efficiency. The speed of response is the same as in basic image processing based on SPDA. The reliability can however be further improved by the application of mobile fire sensors. Increasing fire extinguishing is also possible. It is also can be concluded that intelligent space is a very elegant approach for evacuation purpose. As it is an open platform, the building fire evacuation SDSS can easily be adapted to complex and huge ground or underground buildings, and could be used for crowed situation, such as terminal buildings and subway stations. It is important to note that the application of intelligent space for fire detection and evacuation is very effective, especially in extreme situations and complex building spaces, such as nuclear power station fire. For the low expense of hardware and software requirement this intelligent space can be installed in most public buildings in developing countries.

\section{ACKNOWLEDGEMENTS}

This paper is supported by China's National Science and Technology Support Program (2014BAL05B06)

\section{REFERENCES}

[1] A. K. Gupta and P.K. Yadav. "Safe-r: a new model to study the evacuation profile of a building", Fire Safety Journal, 2004. 39(7): 539556

[2] A. M. Hasofer and I. Thomas. "Analysis of Fatalities and Injuries in Building Fire Statistics”, Fire Safety Journal, 2006. 39(7):2-14.

[3] F. Derbel. "Performance Improvement of Fire Detectors by Means of Gas sensors and Neural Networks", Fire Safety Journal, 2004. 39(5): 383-398. 
[4] F. W. Mowrer and M. G. Pecht, "Exploratory Research on Non-thermal Damage to Electronics form Fires and Fire-Suppression agents," Proceedings of the Annual Reliability and Maintainability Symposium 1995, Washington, DC, USA, 1995, 1-6.

[5] G. Appenzeller, J.H.Lee, H.Hashimoto, "Building Topological Maps by Looking at People: an example of cooperation between intelligent spaces and robots," Proceedings of the 1997 IEEE/RSJ International Conference on Intelligent Robots and Systems (IROS 1997),Vol. 3 Grenobel, France, 1997, 1326-1333.

[6] J. H. Lee, H. Hashimoto, "Intelligent Space, Its Past and Future", The $25^{\text {th }}$ Annual Conference of the IEEE Industrial Electronics Society, Vol. 1, San Joes, CA, USA, 1995, 126-131.

[7] J. H. Lee and H. Hashimoto, "Controlling Mobile Robots in Distributed Intelligent Sensor Network", IEEE Transactions on Industrial Electronics, 2003. 50(5): 890-902.

[8] L. Benthorn and H. Frantzich. "Fire Alarm in a Public Building: how do people evaluate information and choose an evacuation exit?", Fire and Materials, 1999. 23(6): 311-315.

[9] Margrethe Kobes, Ira Helsloot, Bauke de Vries, Jos G. Post. "Building Safety and Human Behaviour in Fire: A Literature Review", Fire Safety Journal. 2010. 45: 1-11.

[10] T. T. Pires, "An Approach for Modeling Human Cognitive Behavior in Evacuation Models", Fire Safety Journal, 2005. 40(2): 177-189.

[11] Wonju Lee, MinkyuCheon, Chang-HoHyun, MignonPark. "Development of building fire safety system with automatic security firm monitoring capability”, Fire Safety Journal, 2013. 58 :65-73.

[12] Y. Gupta and A. Dharmadhikari, "Analysis of False Alarms Given by Automatic Fire Detection Systems", Reliability Engineering, 1985.13(3): 163-174. 